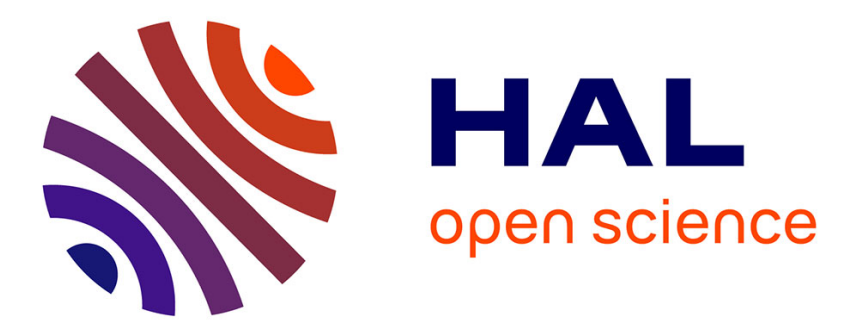

\title{
On the correspondence between risk measures on the space of infinite sequences and their acceptance sets
}

\author{
Elouali Aailal, A. Hassouni
}

\section{To cite this version:}

Elouali Aailal, A. Hassouni. On the correspondence between risk measures on the space of infinite sequences and their acceptance sets. Applied Mathematical Sciences, 2014, 8, pp.4453 - 4458. 10.12988/ams.2014.46479 . hal-03015647

\section{HAL Id: hal-03015647 https://hal.science/hal-03015647}

Submitted on 20 Nov 2020

HAL is a multi-disciplinary open access archive for the deposit and dissemination of scientific research documents, whether they are published or not. The documents may come from teaching and research institutions in France or abroad, or from public or private research centers.
L'archive ouverte pluridisciplinaire HAL, est destinée au dépôt et à la diffusion de documents scientifiques de niveau recherche, publiés ou non, émanant des établissements d'enseignement et de recherche français ou étrangers, des laboratoires publics ou privés. 
Applied Mathematical Sciences, Vol. 8, 2014, no. 90, 4453 - 4458

HIKARI Ltd, www.m-hikari.com

http://dx.doi.org/10.12988/ams.2014.46479

\title{
On the Correspondence between Risk Measures on the Space of Infinite Sequences and their Acceptance Sets
}

\author{
E. Aailal \\ Department of Mathematics and Informatics, Science Faculty \\ University Mohammed V-Agdal, B.P.1014, Rue Ibn Batouta, Rabat, Morocco \\ A. Hassouni \\ Department of Mathematics and Informatics, Science Faculty \\ University Mohammed V-Agdal, B.P.1014, Rue Ibn Batouta, Rabat, Morocco \\ Copyright (c) 2014 E. Aailal and A. Hassouni. This is an open access article distributed \\ under the Creative Commons Attribution License, which permits unrestricted use, distribu- \\ tion, and reproduction in any medium, provided the original work is properly cited.
}

\begin{abstract}
In this paper, we present the correspondence between natural risk statistics on the space of infinite and bounded sequences of real numbers and their associated acceptance sets.
\end{abstract}

Keywords: natural risk statistics, acceptance set, bounded sequence, comonotone convexity

\section{Introduction}

In [2], Artzner and al.(1999), proposed the notion of coherent risk measures for the first time, and associated it with the notion of acceptance sets. So on, the risk measure families that was presented: Insurance risk measures [8].(1997), convex risk measures [5].(2002), and natural risk statistics [6].(2006), still be concerned with defining its corresponding acceptance sets. In [3].(2010), the definition of a natural risk statistics was extended from $\mathbb{R}^{\mathrm{n}}$ to $l^{\infty}(\mathbb{R})$ the space of infinite and bounded sequences of real numbers. We will show that there 
is a correspondence between the set of natural risk statistics on the space $l^{\infty}(\mathbb{R})=\left\{\mathrm{X}=\left(\mathrm{x}_{\mathrm{i}}\right)_{\mathrm{i}=1,2,3, \ldots}: \mathrm{x}_{\mathrm{i}} \in \mathbb{R},\|\mathrm{X}\|_{\infty}=\operatorname{Sup}_{\mathrm{i}=1,2,3, \ldots}\left|\mathrm{x}_{\mathrm{i}}\right|<\infty\right\}$ and the set of acceptance sets in the same space. If $\rho$ is a natural risk statistics on $l^{\infty}$, the acceptance set corresponding to $\rho$ is $A_{\rho}=\left\{X \in l^{\infty}: \rho(X) \leq 0\right\}$. Given $A \subset l^{\infty}$, as an acceptance set, the natural risk statistics corresponding to $\mathrm{A}$, is the map giving every position $X \in l^{\infty}$ the minimal riskless amount that has to be combined with $\mathrm{X}$ in order to obtain an acceptable position, formally we have $\rho_{A}(X)=\operatorname{Inf}\{m \in \mathbb{R}: \mathrm{X}-\mathrm{m} \mathbf{1} \in \mathrm{A}\}$.

\section{Preliminary Notes}

In this section, we recall the definition of a risk measure and an acceptance set, on the space $L_{B}(\Omega, \Gamma)$ of bounded and measurable random variables $[2,5]$.

Definition 2.1 Let $\rho: L_{B}(\Omega, \Gamma) \rightarrow \mathbb{R}$, the function $\rho$ is a risk measure, if: 1- $\rho$ is invariant by translation: $\forall X \in L_{B}(\Omega, \Gamma), \forall c \in \mathbb{R} \rho(X+c)=\rho(X)-c$; 2- $\rho$ is monotone: $\forall X, Y \in L_{B}(\Omega, \Gamma)$ if $X \leq Y$, then $p(Y) \leq p(X)$.

- $\rho$ is convexe if:

3- $\forall \lambda \in[0,1], \forall X, Y \in L_{B}(\Omega, \Gamma) \rho(\lambda X+(1-\lambda) Y) \leq \lambda \rho(X)+(1-\lambda) \rho(Y)$.

- $\rho$ is coherent if:

4- $\rho$ is positively homogeneous: $\forall \lambda \geq 0, \forall X \in L_{B}(\Omega, \Gamma) \rho(\lambda X)=\lambda \rho(X)$;

5- $\rho$ is subadditive: $\forall X, Y \in L_{B}(\Omega, \Gamma)$, we have $\rho(X+Y) \leq \rho(X)+\rho(Y)$.

Definition 2.2 Let $A \subset L_{B}(\Omega, \Gamma), A$ is said to be an acceptance set, if:

$1-A \neq \emptyset$;

2- $A$ is convex;

3- $\forall X \in A, \forall Y \in L_{B}(\Omega, \Gamma)$, if $X \leq Y$, then $Y \in A$.

The next theorem, endows us with a corresponding relationship between convex risk measures on $L_{B}(\Omega, \Gamma)$ and the sets of acceptable positions [2].

Theorem 2.3 ([2]) Let $A \subset L_{B}(\Omega, \Gamma)$ be an acceptance set and $\rho$ a convex risk measure on $L_{B}(\Omega, \Gamma)$, then:

1- $\rho_{A}=\rho$;

2- $A_{\rho}$ is an acceptance set;

3- $\forall X \in A, \forall Y \in L_{B}(\Omega, \Gamma)$, the set $\{\lambda \in[0,1]: \lambda X+(1-\lambda) Y \in A\}$ is closed in $[0,1]$.

Now, we present the definition of a natural risk statistics on $l^{\infty}$, as it was extended in [3], from $\mathbb{R}^{\mathrm{n}}$ to $l^{\infty}$.

Definition 2.4 ([3]) A function $\rho: l^{\infty} \rightarrow \mathbb{R}$ is a natural risk statistics, if: 1- $\rho$ is invariant by translation and positively homogeneous: 
$\rho(\lambda X+c \mathbf{1})=\rho\left(\lambda x_{1}+c, \lambda x_{2}+c, \lambda x_{3}+c, \ldots\right)=\lambda \rho\left(x_{1}, x_{2}, x_{3}, \ldots\right)+c=\lambda \rho(X)+c$ $\forall \lambda \geq 0$ and $\forall c \in \mathbb{R}$, where $\mathbf{1}=(1,1,1, \ldots) \in l^{\infty}$;

2- $\rho$ is monotone: If $X \leq Y$ (i.e., $\left.x_{1} \leq y_{1}, x_{2} \leq y_{2}, x_{3} \leq y_{3}, \ldots\right)$, then $\rho(X)=\rho\left(x_{1}, x_{2}, x_{3}, \ldots\right) \leq \rho(Y)=\rho\left(y_{1}, y_{2}, y_{3}, \ldots\right)$;

3- $\rho$ is comonotone subadditive:

If $X$ and $Y$ are comonotonic (i.e., $\left(x_{i}-x_{j}\right)\left(y_{i}-y_{j}\right) \geq 0$ for any $\left.j \neq i\right)$, then $\rho(X+Y)=\rho\left(x_{1}+y_{1}, x_{2}+y_{2}, \ldots\right) \leq \rho\left(x_{1}, x_{2}, \ldots\right)+\rho\left(y_{1}, y_{2}, \ldots\right)=\rho(X)+\rho(Y)$; 4- $\rho$ is invariant under permutation: for any permutation $\pi=\left(i_{1}, i_{2}, i_{3}, \ldots\right)$ of $\mathbb{N}^{*}$, we have $\rho(X)=\rho\left(x_{1}, x_{2}, x_{3}, \ldots\right)=\rho\left(x_{i_{1}}, x_{i_{2}}, x_{i_{3}}, \ldots\right)=\rho(\pi(X))$.

Note that every natural risk statistics $\rho$ is normalized (i.e., $\rho(0)=0$ ) and Lipschitz continuous with respect to the usual supremum norm of $l^{\infty}$, then it is continuous.

\section{Main Results}

The next definition is the analogue in $l^{\infty}$, of the definition $2.2[2,5]$.

Definition 3.1 Let $A \subset l^{\infty}, A$ is said to be an acceptance set, if:

1- $A$ contains the set $l_{-}^{\infty}=\left\{X \in l^{\infty}: x_{i} \leq 0, i=1,2,3, \ldots\right\}$;

2- $A \cap l_{++}^{\infty}=\left\{X \in l^{\infty}: x_{i}>0, i=1,2,3, \ldots\right\}=\emptyset$;

3- $A$ is comonotone convex: If $X$ and $Y$ are comonotonic, then $(\lambda X+(1-\lambda) Y) \in A, \forall \lambda \in[0.1]$

4- $A$ is positively homogeneous: $\lambda X \in A, \forall X \in A$ and $\forall \lambda \geq 0$;

5- $\forall X, Y \in l^{\infty}$, If $X \leq Y$ and $Y \in A$, then $X \in A$;

6- $\pi(X) \in A \subset l^{\infty}$, for every permutation $\pi(X)$ of $X \in A$.

Remark 3.2 The difference in inequalities and signs between this definition and the definition 2.2 in [2] and [5], is that here we consider that $X$ is a loss, as in [3], contrary to [2] and [5] where $X$ is considered as a profit.

Now, we are in a position to state the correspondence theorem, between a natural risk statistics on $l^{\infty}$ and its associated acceptance set.

Theorem 3.31 - If $\rho$ is a natural risk statistics on $l^{\infty}$, then the set $A_{\rho}=\left\{X \in l^{\infty}: \rho(X) \leq 0\right\}$ is a closed acceptance set in the topology of the usual supremum norm of $l^{\infty}$.

2- If a set $A \subset l^{\infty}$ is an acceptance set, then $\rho_{A}(X)=\operatorname{Inf}\{m \in \mathbb{R}: \mathrm{X}-\mathrm{m} \mathbf{1} \in \mathrm{A}\}$ is a natural risk statistics on $l^{\infty}$.

Proof. $1 \bullet \forall X \in l_{-}^{\infty}$, we have $x_{i} \leq 0, i=1,2,3, \ldots$, so $X \leq 0$, the monotonicity of $\rho$ implies that $\rho(X) \leq \rho(0)=0$, then $X \in A_{\rho}$. Therefore, $l_{-}^{\infty} \subset A_{\rho}$. 
- Let $X \in l_{++}^{\infty}$, so $X>0$ and $\rho(X) \geq \rho(0)=0$. If $\rho(X)>0$, then $X \notin A_{\rho}$. If $\rho(X)=0$, then $\forall Y$ such that $0<Y \leq X<\mathbf{1}$, we have $0 \leq \rho(Y) \leq \rho(X)=0$, thus $\rho(Y)=0$. Let $B=\left\{Z \in l_{++}^{\infty}: X<Z<\mathbf{1}, 0<\rho(Z), 0<\operatorname{Inf}(Z)\right\}$, by continuity of $\rho$ we obtain that $\operatorname{In} f(B)=X$, then $\forall \epsilon>0, \exists Z \in B$ such that $Z<X+\epsilon \mathbf{1}$, then $Z-\epsilon \mathbf{1}<X$, we choose $\epsilon=\operatorname{Inf}\left(Z^{\prime}\right)$ where $0<Z^{\prime}<Z$, then $0<Z^{\prime}-\operatorname{In} f\left(Z^{\prime}\right) \mathbf{1}<Z-\operatorname{Inf}\left(Z^{\prime}\right) \mathbf{1}<X$, hence $\rho\left(Z^{\prime}-\operatorname{Inf}\left(Z^{\prime}\right) \mathbf{1}\right)=0$ and $\rho\left(Z^{\prime}\right)=\operatorname{Inf}\left(Z^{\prime}\right)$, which is impossible for $Z^{\prime}$ non constant. Therefore, $\rho(X)>0$, and $X \notin A_{\rho}$. Finally, $l_{++}^{\infty} \bigcap A_{\rho}=\emptyset$.

- If $\mathrm{X}$ and $\mathrm{Y}$ are comonotonic, where $X \in A_{\rho}$ and $Y \in A_{\rho}$, then $\rho(X) \leq 0$ and $\rho(Y) \leq 0$, in another side $\lambda X$ and $(1-\lambda) Y$ are comonotonic $\forall \lambda \in[0,1]$. Thus, the comonotonic subadditivity and the positive homogeneity of $\rho$, imply that $\rho(\lambda X+(1-\lambda) Y) \leq \rho(\lambda X)+\rho((1-\lambda) Y)=\lambda \rho(X)+(1-\lambda) \rho(Y) \leq 0$, then $(\lambda X+(1-\lambda) Y) \in A_{\rho}$. Hence, $A_{\rho}$ is comonotonic convex.

- $\forall X \in A_{\rho}$, we have $\rho(X) \leq 0 . \forall \lambda \geq 0$, the positive homogeneity of $\rho$ implies that $\rho(\lambda X)=\lambda \rho(X) \leq 0$, then $\lambda X \in A_{\rho}$. Therefore, $A_{\rho}$ is positively homogeneous.

- $\forall X, Y \in l^{\infty}$, If $X \leq Y$ and $Y \in A_{\rho}$, then $\rho(Y) \leq 0$. By monotonicity of $\rho$, we have $\rho(X) \leq \rho(Y) \leq 0$, then $X \in A_{\rho}$. Hence, every element of $l^{\infty}$ less than $\mathrm{Y}$, is in $A_{\rho}$.

- If $X \in A_{\rho}$, then $\rho(X) \leq 0$. Let $\pi=\left(i_{1}, i_{2}, i_{3}, \ldots\right)$ be a permutation of $\mathbb{N}^{*}$. By invariance under permutation of $\rho$, we have

$\rho(\pi(X))=\rho\left(x_{i_{1}}, x_{i_{2}}, x_{i_{3}}, \ldots\right)=\rho(X)=\rho\left(x_{1}, x_{2}, x_{3}, \ldots\right) \leq 0$, then $\pi(X) \in A_{\rho}$. Therefore, $A_{\rho}$ contains the permutations of its elements.

- If $\left(X^{k}\right)_{k \in \mathrm{N}^{*}}$ is a sequence in $A_{\rho}$, converges in the norm $\|\cdot\|_{\infty}$ to $X=\left(x_{1}, x_{2}, x_{3}, \ldots\right) \in l^{\infty}$, we have $\rho\left(X^{k}\right) \leq 0, \forall k=1,2,3, \ldots$ If $X \notin A_{\rho}$, then $\rho(X)>0$. Thus $\exists \epsilon>0$ such that $\rho(X-\epsilon \mathbf{1})>0$. From the fact that $\lim _{k \rightarrow+\infty} X^{k}=X$, it follows that $\exists k_{0} \in \mathbb{N}^{*}$ such that $X-\epsilon \mathbf{1}<X^{k_{0}}$. By monotonicity of $\rho$, we have $0<\rho(X-\epsilon \mathbf{1}) \leq \rho\left(X^{k_{0}}\right)$, which contradicts the fact that $X^{k_{0}}$ is an element of $A_{\rho}$. Finally, we have $X \in A_{\rho}$ and $A_{\rho}$ is closed in the topology of the supremum norm of $l^{\infty}$.

2 • Firstly, $\rho_{A}(X)=\operatorname{In} f\{m \in \mathbb{R}: \mathrm{X}-\mathrm{m} \mathbf{1} \in \mathrm{A}\}$ is well defined, we have $l_{-}^{\infty} \subset A$, so $A \neq \emptyset$. For $Y \in A Y-\operatorname{Inf}(Y) \mathbf{1}=Y-\operatorname{Inf}\left\{y_{i}, i=1,2,3, \ldots\right\} \mathbf{1} \geq 0$ and $\forall X \in l^{\infty}$, we have $X-\operatorname{Sup}(X) \mathbf{1}=X-\operatorname{Sup}\left\{x_{i}, i=1,2,3, \ldots\right\} \mathbf{1} \leq 0$, so $X+(\operatorname{In} f(Y)-\operatorname{Sup}(X)) \mathbf{1} \leq Y$, which means that $X+(\operatorname{Inf}(Y)-\operatorname{Sup}(X)) \mathbf{1} \in A$, then $\operatorname{Sup}(X)-\operatorname{Inf}(Y) \in\{m \in \mathbb{R}: \mathrm{X}-\mathrm{m} \mathbf{1} \in \mathrm{A}\}$.

Thus, $\{m \in \mathbb{R}: \mathrm{X}-\mathrm{m} \mathbf{1} \in \mathrm{A}\} \neq \emptyset$.

Also, $\rho_{A}(X)=\operatorname{Inf}\{m \in \mathbb{R}: \mathrm{X}-\mathrm{m} \mathbf{1} \in \mathrm{A}\}>-\infty$, because $\mathrm{X}$ is bounded $(\operatorname{In} f(X)-m) \mathbf{1} \leq X-m \mathbf{1}$, so if $X-m \mathbf{1} \in A$, then $(\operatorname{In} f(X)-m) \mathbf{1} \in A$, which means that $(\operatorname{Inf}(X)-m) \mathbf{1} \in l_{-}^{\infty}$, then $\operatorname{Inf}(X) \leq m$. Therefore, $\rho_{A}$ exists.

- Let $X \in l^{\infty}$ and $c \in \mathbb{R}$, we have $\rho_{A}(X+c \mathbf{1})=\operatorname{In} f\{m \in \mathbb{R}: \mathrm{X}+\mathrm{c} \mathbf{1}-\mathrm{m} \mathbf{1} \in \mathrm{A}\}$ $=\mathrm{c}+\operatorname{Inf}\{\mathrm{m} \in \mathbb{R}: \mathrm{X}-\mathrm{m} \mathbf{1} \in \mathrm{A}\}=\mathrm{c}+\rho_{\mathrm{A}}(\mathrm{X})$. Let $\lambda \geq 0$. If $\lambda=0$, then we 
have $\rho_{A}(\lambda X)=\operatorname{Inf}\{m \in \mathbb{R}:-\mathrm{m} \mathbf{1} \in \mathrm{A}\}=\lambda \rho_{\mathrm{A}}(\mathrm{X})=0$, because $l_{++}^{\infty} \bigcap A=\emptyset$ and $l_{-}^{\infty} \subset A$. If $\lambda>0$, then $\rho_{A}(\lambda X)=\operatorname{Inf}\{m \in \mathbb{R}: \lambda \mathrm{X}-\mathrm{m} 1 \in \mathrm{A}\}$ $=\lambda \operatorname{Inf}\{\mathrm{v} \in \mathbb{R}: \lambda(\mathrm{X}-\mathrm{v} \mathbf{1}) \in \mathrm{A}\}=\lambda \operatorname{Inf}\{\mathrm{v} \in \mathbb{R}:(\mathrm{X}-\mathrm{v} \mathbf{1}) \in \mathrm{A}\}=\lambda \rho_{\mathrm{A}}(\mathrm{X})$, because $\mathrm{A}$ is positively homogeneous. Therefore, $\rho_{A}$ is positively homogeneous and invariant by translation.

- $\forall X, Y \in l^{\infty}$, if $X \leq Y$ and $Y-m \mathbf{1} \in A$, then $X-m \mathbf{1} \in A$ as an element of $l^{\infty}$ less than $Y-m \mathbf{1}$ and $\{m \in \mathbb{R}: \mathrm{Y}-\mathrm{m} \mathbf{1} \in \mathrm{A}\} \subset\{\mathrm{m} \in \mathbb{R}: \mathrm{X}-\mathrm{m} \mathbf{1} \in \mathrm{A}\}$. Hence, $\rho_{A}(Y)=\operatorname{Inf}\{m \in \mathbb{R}: \mathrm{Y}-\mathrm{m} \mathbf{1} \in \mathrm{A}\} \geq \rho_{\mathrm{A}}(\mathrm{X})=\operatorname{Inf}\{\mathrm{m} \in \mathbb{R}: \mathrm{X}-\mathrm{m} \mathbf{1} \in \mathrm{A}\}$, which means that $\rho_{A}$ is monotone.

- Let $X, Y \in l^{\infty}$ be comonotonic. If $m, m^{\prime} \in \mathbb{R}$ are such that $X-m \mathbf{1} \in A$ and $Y-m^{\prime} \mathbf{1} \in A$, then from the fact that $X-m \mathbf{1}$ and $Y-m^{\prime} \mathbf{1}$ are comonotonic and $\mathrm{A}$ is comonotone convex, it follows that $\frac{1}{2}(X-m \mathbf{1})+\frac{1}{2}\left(Y-m^{\prime} \mathbf{1}\right) \in A$. By positive homogeneity of $\mathrm{A}$, we obtain $X+Y-\left(m+m^{\prime}\right) \mathbf{1} \in A$. Thus, $\rho_{A}(X+Y) \leq m+m^{\prime}$, especially

$\rho_{A}(X+Y) \leq \operatorname{Inf}\{m \in \mathbb{R}: \mathrm{X}-\mathrm{m} \mathbf{1} \in \mathrm{A}\}+\operatorname{Inf}\left\{\mathrm{m}^{\prime} \in \mathbb{R}: \mathrm{Y}-\mathrm{m}^{\prime} \mathbf{1} \in \mathrm{A}\right\}$, which means that $\rho_{A}(X+Y) \leq \rho_{A}(X)+\rho_{A}(Y)$. Therefore, $\rho_{A}$ is comonotone subadditive.

- $\forall \pi=\left(i_{1}, i_{2}, i_{3}, \ldots\right)$ a permutation of $\mathbb{N}^{*},(\pi(X)) \in A, \forall X \in A$, so $\forall m \in \mathbb{R}$ we have $(X-m \mathbf{1}) \in A$ if and only if $(\pi(X)-m \mathbf{1}) \in A$, thus

$\{m \in \mathbb{R}: \mathrm{X}-\mathrm{m} \mathbf{1} \in \mathrm{A}\}=\{\mathrm{m} \in \mathbb{R}: \pi(\mathrm{X})-\mathrm{m} \mathbf{1} \in \mathrm{A}\}$, so

Inf $\{m \in \mathbb{R}: \mathrm{X}-\mathrm{m} \mathbf{1} \in \mathrm{A}\}=\operatorname{Inf}\{\mathrm{m} \in \mathbb{R}: \pi(\mathrm{X})-\mathrm{m} \mathbf{1} \in \mathrm{A}\}$. Therefore, $\rho_{A}(X)=\rho_{A}(\pi(X))$.

The next corollary shows that the set of acceptable positions associated with the natural risk statistics defined by an acceptance set, is the closure of the set itself.

Corollary 3.4 Let $C \subset l^{\infty}$ be an acceptance set, we have $A_{\rho_{C}}=\bar{C}$, where $\bar{C}$ is the closure of $C$ in the topology of the supremum norm of $l^{\infty}$.

Proof. If $X \in C$, then $\rho_{C}(X) \leq 0$, thus $X \in A_{\rho_{C}}$, which means that $C \subset$ $A_{\rho_{C}}$. According to the theorem $3.3, A_{\rho_{C}}$ is a closed set in the topology of the supremum norm of $l^{\infty}$, so $\bar{C} \subset A_{\rho_{C}}$.

$\forall X \in A_{\rho_{C}}$ we have $\rho_{C}(X)=\operatorname{Inf}\{m \in \mathbb{R}: \mathrm{X}-\mathrm{m} 1 \in \mathrm{C}\} \leq 0$.

If $\operatorname{Inf}\{m \in \mathbb{R}: \mathrm{X}-\mathrm{m} \mathbf{1} \in \mathrm{C}\}<0$, then $\exists m<0$ such that $X-m \mathbf{1} \in C$, since $\mathrm{X}$ is an element of $l^{\infty}$ such that $X<X-m \mathbf{1}$, then $X \in C \subset \bar{C}$.

If $\operatorname{In} f\{m \in \mathbb{R}: \mathrm{X}-\mathrm{m} \mathbf{1} \in \mathrm{C}\}=0$, then $\exists\left(m_{i}\right)_{i=1,2,3, \ldots}$ a sequence of real positives such that $\lim _{i \rightarrow+\infty} m_{i}=0$ and $X-m_{i} \mathbf{1} \in C$, so $\lim _{i \rightarrow+\infty}\left(X-m_{i} \mathbf{1}\right)^{i}=X$ in the supremum norm of $l^{\infty}$.

Thus, $X \in \bar{C}$ and $A_{\rho_{C}} \subset \bar{C}$. Finally, we have $A_{\rho_{C}}=\bar{C}$.

Now we explicit the natural risk statistics, associated with $A_{\rho}$. 
Proposition 3.5 If $\rho$ is a natural risk statistics on $l^{\infty}$, then

$\forall X \in l^{\infty} \rho(X)=\rho_{A_{\rho}}(X)=\operatorname{Inf}\left\{m \in \mathbb{R}: \mathrm{X}-\mathrm{m} \mathbf{1} \in \mathrm{A}_{\rho}\right\}$.

Proof. $\forall X=\left(x_{1}, x_{2}, x_{3}, \ldots\right) \in l^{\infty}$, we have by translation invariance of $\rho$ $\rho_{A_{\rho}}(X)=\operatorname{Inf}\left\{m \in \mathbb{R}: \mathrm{X}-\mathrm{m} \mathbf{1} \in \mathrm{A}_{\rho}\right\}=\operatorname{Inf}\{\mathrm{m} \in \mathbb{R}: \rho(\mathrm{X}-\mathrm{m} \mathbf{1}) \leq 0\}$

$=\operatorname{Inf}\{\mathrm{m} \in \mathbb{R}: \rho(\mathrm{X}) \leq \mathrm{m}\}=\rho(\mathrm{X})$. So, $\rho=\rho_{A_{\rho}}$.

\section{References}

[1] S. Ahmed, D. Filipovic, G. Svindland, A note on natural risk statistics , Operations Research Letters, 36 (2008), 662 - 664.

[2] P. Artzner, F. Delbaen, J.-M. Eber, D. Heath, Coherent measures of risk, Mathematical Finance, 9(3) (1999), 203 - 228.

[3] H. Assa, M. Morales, Risk measures on the space of infinite sequences, Mathematics and Financial Economics, 2(4) (2010), 253 - 275.

[4] J. Dhaene, S. Vanduffel, M.J. Goovaerts, R. Kaas, Q. Tang, D. Vyncke, Risk measures and comonotonicity: a review, Stochastic Models, 22(4) (2006), $573-606$.

[5] H. Föllmer, A. Schied, Convex measures of risk and trading constraints, Finance Stoch, 6 (4) (2002), 429 - 447.

[6] C. C. Heyde, S. G. Kou, X. H. Peng, What is a good risk measure: Bridging the gaps between date,coherent risk measures, and insurance risk measures, Working paper, Columbia University, (2006).

[7] D. Tian, X. Suo, A note on convex risk statistics, Operations Research Letters, 40(2012), $551-553$.

[8] S.S. Wang, V.R. Young, H.H. Panjer, Axiomatic characterization of insurance prices, Insurance: Mathematics and Economics, 21 (1997), 173 183.

Received: June 7, 2014 